\title{
Trabajando juntos: hacia un modelo común de evaluación de la formación en competencias informacionales
}

\author{
Working together towards a common assessment model for information literacy development in Spanish universities
}

\section{Isabel Casal (1), Elena Escar (2), Maria Esteban (3), Carmen Julia Hernández (4), Gloria SerRano} (5), Carmen VARELA-PRADo (6)

(1) Universidad de Alicante, maria.esteban@ua.es, $(2,3)$ Universidad de Zaragoza, eescar@unizar.es, gserrano@unizar.es , (5) Universidad de La Laguna, cjhdez@ull.es, (6) Universidad de Santiago, carmen.varela@usc.es

\begin{abstract}
Resumen
Se presenta un proyecto de trabajo compartido entre cuatro universidades: La Laguna, Zaragoza, Alicante y Santiago de Compostela alrededor de un modelo común de formación en competencias informacionales. Para ello, comenzamos contextualizando el proyecto, describiendo el modelo de curso establecido en el convenio de cooperación, analizamos su puesta en marcha en el curso académico 2010/2011 y los resultados obtenidos de manera individual y global, exponemos el método de trabajo seguido y finalizamos planteando los retos de futuro.
\end{abstract}

Palabras clave: Competencias informacionales. Alfabetización informacional. e-Learning. Cooperación interbibliotecaria.

\section{Introducción}

El marco de reflexión creado alrededor del nuevo Espacio Europeo de Educación Superior ofreció la oportunidad de definir conjuntamente los conocimientos y las competencias que los estudiantes universitarios deben adquirir. Muchos estudios apuntan a que el éxito de este nuevo modelo de aprendizaje requiere del alumnado la adquisición de competencias en información, es decir, que el estudiante sea capaz de localizar, organizar, evaluar, utilizar y comunicar eficazmente información.

La responsabilidad de integrar estas competencias en el itinerario educativo recae en la propia universidad, y supone la idea de llevar la biblioteca al aula y de estrechar el vínculo de la docencia con los recursos de información.

Si desarrollar el aprendizaje a lo largo de la vida de los estudiantes constituye uno de los objetivos principales de las instituciones de educación superior, la alfabetización informacional se convierte de esta manera en una de las claves dentro de este proceso de aprendizaje. En estas circunstancias, las y los universitarios deben incorporar esta competencia para aprender a

\begin{abstract}
A project of four Spanish universities (La Laguna, Zaragoza, Alicante and Santiago de Compostela) to develop a common model for information skills training is presented. The project is contextualized, the current model, established in the agreement for cooperation, is detailed, and the results of its implementation in the 2010/2011 academic year are discussed.
\end{abstract}

Keywords: Information skills. Information literacy. Library cooperation. e-Learning.

interaccionar y gestionar la información con eficacia tanto en el ámbito académico como en el laboral e incorporarla a su proceso de estudio como herramienta necesaria para el trabajo de aprender (Varela Prado, 2005; USC, 2010)

En el marco de REBIUN, sectorial de la CRUE dedicada a las Bibliotecas universitarias, se ha venido reflexionando y trabajando sobre este tema a lo largo de los últimos años. Así se ha considerado que una de las estrategias de futuro de las bibliotecas universitarias como servicio básico de apoyo a la investigación, la docencia y el aprendizaje es la de potenciar la colaboración en una oferta educativa en capacitación informacional para el alumnado de los nuevos grados, contribuyendo de esta manera a los objetivos de la institución, entre los que se encuentra el dar una adecuada respuesta a esta sociedad en permanente transformación.

En el contexto de las bibliotecas de la red REBIUN, surge como iniciativa de la biblioteca universitaria de la Universidad de La Laguna (ULL) proponer a las bibliotecas Universitarias de Zaragoza (UZ) y Santiago de Compostela (USC) firmar un convenio de colaboración para desarrollar un modelo común de curso de inicia- 
ción en formación de competencias informacionales para alumnos de primero de Grado. Posteriormente se ha incorporado la Universidad de Alicante.

El grupo ULL/USC/UZ firma el convenio de cooperación en Junio de 2010 (1) teniendo presente que las bibliotecas universitarias, ante el reto del Espacio Europeo de Educación Superior y el cambio de concepción del modelo de enseñanza/aprendizaje, quieren apoyar a los profesores y a los estudiantes en la adquisición de competencias informacionales, a través de cursos de formación. Las bibliotecas de estas universidades están interesadas en desarrollar un modelo común de curso sobre competencias informacionales para los estudiantes de grado (primer curso) con el objetivo de mejorar la formación ofertada por dichos servicios y tener una evaluación común del aprendizaje de los alumnos en las diferentes universidades.

Al objetivo inicial de formar en competencias informacionales, se ha ido incorporando la idea de formar, de manera progresiva, según las posibilidades de cada biblioteca, en competencias informáticas, por lo menos en aspectos básicos relacionados con las TIC.

\section{Cuatro realidades en un mismo marco educativo: el objeto de la cooperación}

Se crea esta pequeña red de trabajo cooperativo con la adopción y puesta en marcha en las cuatro universidades del programa de la universidad de La Laguna, destacando en la agenda la voluntad de definir y avanzar en un plan común de investigación en competencias informacionales, producir estudios cooperativos e integrados en esta red, con datos comparables, promover esta formación e intercambiar experiencias que se puedan externalizar como ejemplo de buenas prácticas.

\subsection{Aspectos que se tuvieron en cuenta}

- Oportunidad: La Biblioteca de la Universidad de La Laguna cede un modelo de curso.

- Eficacia: ahorro de esfuerzo (todos hacemos lo mismo).

- Apoyo institucional: Los rectores apoyan la incorporación de la competencia informacional a través de la firma del documento.

- Forma de trabajo: A través de la colaboración logramos un mejor modelo.

- Evaluación: Un único modelo de formación que evalúa a un mayor número de universitarios.
El objetivo inicial es elaborar un informe acerca de las competencias en información que posee el alumnado y establecer un diagnóstico sobre la situación actual, con datos comparables entre las cuatro universidades, y mejorar las propuestas de formación.

\subsection{Objetivos generales}

Los objetivos generales del curso son:

- Que el alumnado se inicie en la adquisición de las habilidades y destrezas básicas para plantear una búsqueda de información, localizar, seleccionar, analizar y construir nuevos conocimientos de forma autónoma, constructiva y ética de acuerdo con sus necesidades o problemas informacionales.

- Que el alumnado asuma que saber gestionar información es una práctica que debe completar su proceso de formación en la universidad, que le facilita el estudio y la realización de trabajos académicos con metodología científica, y que tiene que constituir una práctica transferible a todo su trabajo formativo, investigador, profesional y cotidiano.

- Que el alumnado sea capaz de conseguir estos objetivos a través de una plataforma de enseñanza/aprendizaje virtual, donde se da importancia a herramientas de cooperación, autoaprendizaje y utilización de TIC.

\subsection{Elementos esenciales del modelo formativo}

A) El curso se imparte en modalidad virtual (2) en las cuatro universidades y preferentemente en el primer cuatrimestre del curso académico, ya que es una actividad para trabajar una competencia transversal, genérica y necesaria desde el primer día de universidad. La responsabilidad de la gestión corre a cargo de dos coordinadores por cada universidad y los tutores correspondientes por curso.

El curso es el resultado de la colaboración entre el Servicio de Biblioteca y el profesorado de $1^{\circ}$ de grado. Se persigue que el curso sea incluido en la guía docente de una asignatura obligatoria de grado (3), porque las asignaturas obligatorias son las que tienen el contenido específico de la titulación, ya que responden a las competencias específicas de ese título. Este objetivo ya se ha conseguido con éxito en La Laguna, al llevar más años de trabajo en esta materia.

B) La evaluación de la formación se centra en dos aspectos fundamentalmente:

- El grado de aprendizaje de los alumnos en el curso y el aprendizaje posterior a través de 
indicadores que se le aportarán a los profesores colaboradores para la comprobación del aprendizaje.

- El grado de satisfacción de los alumnos, de los profesores colaboradores y el de los bibliotecarios formadores. Este elemento ayuda a mejorar el enfoque y los contenidos del curso adaptándolos a las necesidades de docentes y discentes.

C) De la recogida y explotación de datos de las cuatro experiencias, se extraen los datos parciales por universidad y los globales de las cuatro. Los datos recogidos y evaluados aportan la información que nos permite estar en condiciones de establecer en conjunto propuestas de mejora en cuanto a materiales didácticos, unidades temáticas, tareas y encuestas.

D) Desde el principio se señala la importancia de la difusión de esta iniciativa, para lo que se detalla un programa específico de difusión de la misma. El objetivo es demostrar que no hay problemas de distancia, ni con las distintas políticas o filosofías de cada universidad, sino que es un proyecto que hemos afrontado con actitud positiva confiando en el aprendizaje compartido y que pretendemos externalizar como modelo de benchmarking.

E) Cada una de las Universidades asume la coordinación de un aspecto del modelo formativo: materiales didácticos (Alicante), contenidos (La Laguna), evaluación (Zaragoza) y difusión (Santiago de Compostela).

\section{El modelo del curso: descripción}

El curso está concebido para que el alumno le dedique entre 5 y 10 horas, dependiendo de las competencias previas que tenga.

En el curso el alumno aprende a:

- Identificar la necesidad de información

- Localizar y seleccionar información

- Evaluar la información encontrada

- Usar de forma ética la información

- Comunicar de forma adecuada la información

- Utilizar de manera adecuada las TIC

El curso se estructura a través de una serie de unidades, cada unidad incorpora objetivos, material didáctico y tareas. Éstas últimas permiten al alumnado comprobar su aprendizaje.

Es el mismo curso para las diferentes titulaciones de grado, a excepción de los temas de las prácticas, las normas de cita, los recursos y bibliografía recomendada que se adecúan a cada grado. Estos dos últimos elementos son recursos de apoyo, no unidades dentro de los cursos.

El curso integra herramientas que ayudan al alumno a trabajar de forma cooperativa, a través de los foros de debate. En estos foros el bibliotecario y/o el profesor colaborador, introducen una serie de temas de discusión relacionados con el curso y/o la asignatura de la titulación donde se ha integrado.

Además, el curso tiene otros foros que sirven para establecer una comunicación permanente, para aclarar dudas o hacer sugerencias.

El alumnado comienza el curso contestando un cuestionario inicial que sirve para valorar el nivel de competencias que tiene.

Las unidades del curso se han diseñado para que el alumnado las realice de forma secuencial y son las siguientes:

0 . Antes de empezar: la importancia de adquisición de competencias

1. El proceso de búsqueda de información

2. Cómo localizar información en el catálogo

3. Cómo localizar información electrónica en la web de la biblioteca

4. Cómo localizar información en Internet

5. Cómo citar la información localizada

6. Investigar en $1^{\circ}$ de grado: hacer un trabajo

7. La web 2.0

Recientemente se han establecido las unidades mínimas que debe tener el curso, que son las que serán evaluadas por todas las bibliotecas integrantes del Convenio.

Cada biblioteca readapta los prácticos (algunas han elegido que sean tipo test) y algunos materiales didácticos conforme a sus titulaciones, recursos y plataformas de información disponibles.

El curso tiene una serie de materiales didácticos (guías, tutoriales, vídeos...) que son comunes y otros que cada biblioteca integra para mejorar el aprendizaje.

Al finalizar el curso el alumnado debe cumplimentar una encuesta final que le sirve para comprobar lo aprendido; en ella, se incorporan ítems relacionados con cada unidad e ítems del cuestionario inicial para valorar la evolución del aprendizaje. 


\section{Análisis de resultados}

Uno de los objetivos del proyecto es la evaluación de los cursos y del aprendizaje de los alumnos (4). Contamos para ello con una línea de trabajo dedicada al análisis de resultados, con el objetivo de establecer un sistema de indicadores que nos permita contar, a corto y medio plazo, con un sistema de seguimiento, medición, análisis y mejora del modelo de curso para alumnos de primero de grado en el marco del convenio de cooperación.

Se presentan los resultados de esta primera implantación del modelo de curso en las cuatro universidades. Hemos de tener en cuenta que el punto de partida en cuanto a situación y realidad de las cuatro universidades participantes en el proyecto es distinto, y que deberemos ir adaptando el sistema de indicadores a las situaciones y realidades de nuestras respectivas universidades, asegurando la eficacia y eficiencia del mismo (Tablas I y II).

\begin{tabular}{lrrrr}
\hline Universidad & Grados & Profesores & Alumnos & Nuevos \\
\hline Alicante & 39 & 2.387 & 29.755 & 6.782 \\
\hline La Laguna & 41 & 1.716 & 24.744 & 5.053 \\
\hline Santiago & 48 & 2.302 & 29.305 & 4.508 \\
\hline Zaragoza & 54 & 3.715 & 30.478 & 7.683 \\
\hline
\end{tabular}

Tabla I. Datos de situación curso académico 2010/11

6.243 alumnos han realizado el curso

El $88 \%$ de los alumnos lo han superado con éxito

74 bibliotecarios han participado en el proyecto

El $85 \%$ de los alumnos dice que ha adquirido nuevos conocimientos

El $80 \%$ de los alumnos creen que el curso les servirá en su formación académica

El 82\% de los alumnos recomendarían el curso a otros estudiantes

Tabla II. Resultados globales del curso 2010/11

En esta primera fase de implementación del proyecto se han seleccionado para el análisis comparativo un total de cinco grandes bloques de indicadores:

1. Para comparar la integración del curso en las titulaciones.

2. Para comparar datos de alumnado de grado formado en $\mathrm{Cl}$.

3. Para comparar datos de personal bibliotecario formador en $\mathrm{Cl}$.
4. Para comparar la satisfacción con el curso de los distintos agentes.

5. Para comparar resultados de la evaluación de aprendizaje y eficacia de la formación en $\mathrm{Cl}$.

4.1. Indicadores para medir la integración de esta actividad de aprendizaje en las titulaciones

- Porcentaje de grados en los que se imparte el curso respecto al total de grados que se imparten en la Universidad (gráfico I)

- Porcentaje de grados con curso incorporado en la guía docente del total de grados en los que se imparte el curso (gráfico II)

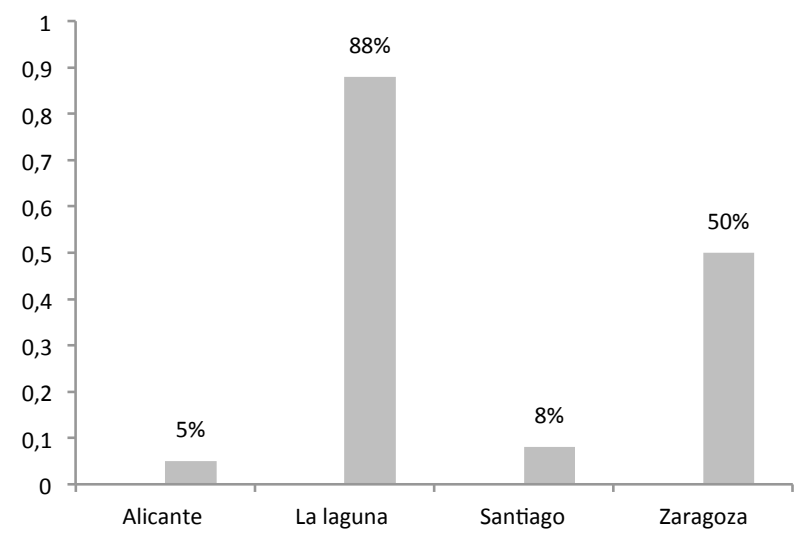

Gráfico I. Integración del curso en los grados

La Universidad de La Laguna viene aplicando este modelo de formación desde el curso 2006/2007. El resto de las universidades lo han implantado el curso 2010/2011.

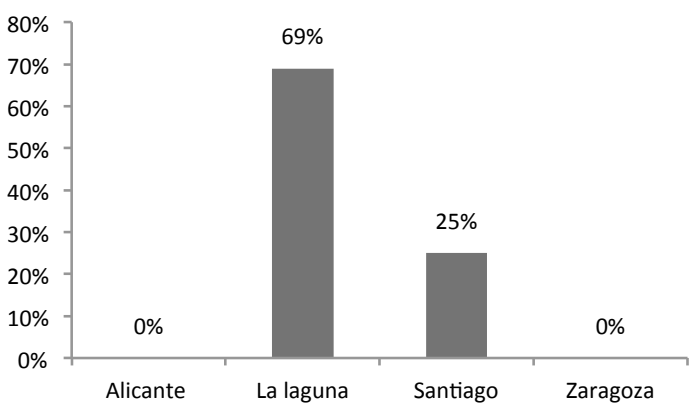

Gráfico II. Integración del curso en las titulaciones: guías docentes

En cuanto a la tasa de inclusión, en este curso académico sólo la ULL y la USC tienen incorporada esta actividad de aprendizaje en las guías docentes de las asignaturas en las que se integra (en un 69 y un $25 \%$ de los casos, respecti- 
vamente). Es objetivo para el curso 2011/12 que todos los grados en los que se integra el curso lo reflejen en las guías docentes de las asignaturas de primer curso con la que se colabora.

\subsection{Indicadores para medir los resultados}

- Porcentaje de alumnos matriculados en los cursos del total de alumnos de nuevo ingreso (grafico III).

- Porcentaje de alumnos aptos del total alumnos inscritos en los cursos (tasa rendimiento) (gráfico V).

- Porcentaje de alumnos aptos del total alumnos que han realizado el curso (tasa de éxito) (gráfico VI).

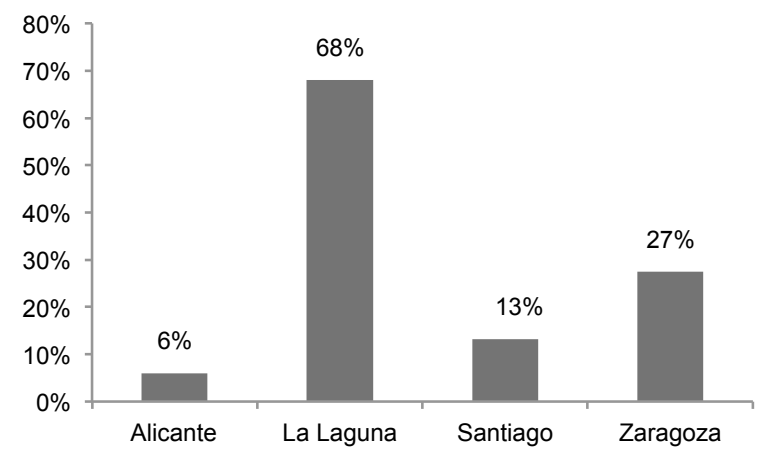

Gráfico III. Alumnos que realizan el curso sobre el total de nuevo ingreso

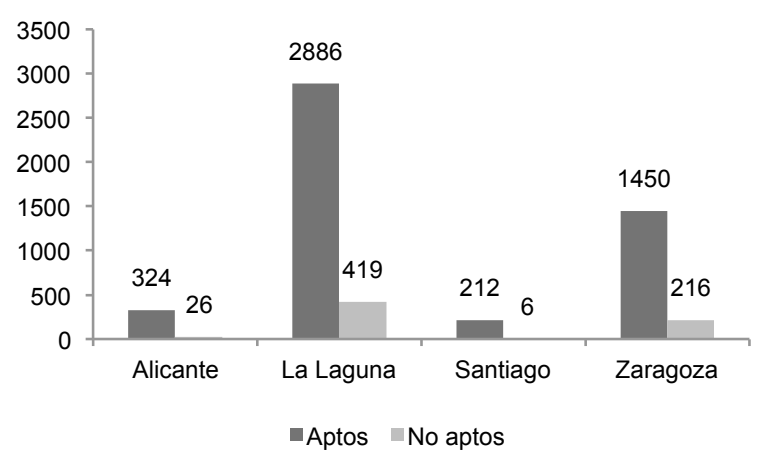

Gráfico IV. Alumnos que superan el curso

Se presentan los datos del porcentaje de los alumnos que entraron en septiembre de 2010 en cada universidad que han tenido la oportunidad de realizar el curso sobre el total de alumnos inscritos en los cursos (5).

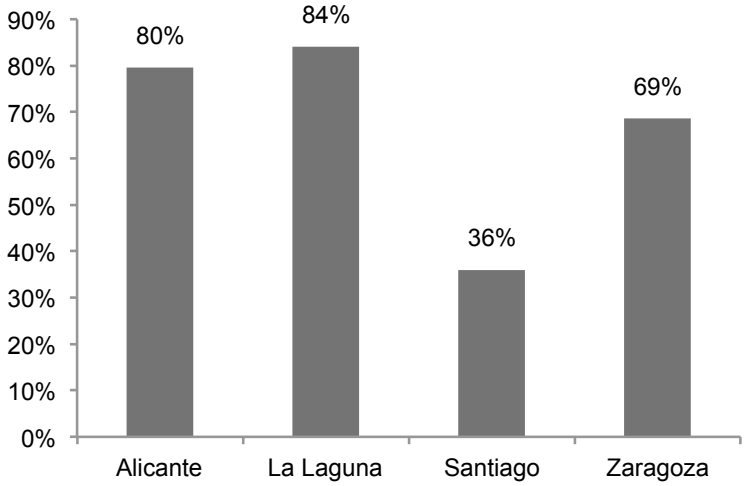

Gráfico V. Porcentaje de alumnos aptos

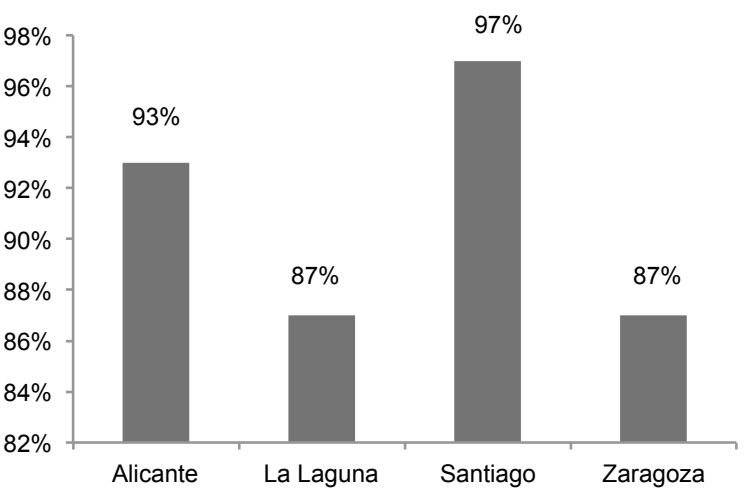

Gráfico VI. porcentaje de alumnos aptos sobre el total alumnos que realizan el curso

4.3. Indicadores para medir el aprendizaje de los alumnos

Objetivo prioritario para el curso $11 / 12$, es contar con datos de evaluación del aprendizaje de los alumnos. Para ello se ha diseñado un cuestionario mediante el cual el profesor valorará el nivel de aplicación en los trabajos de clase de lo aprendido por sus alumnos.

\subsection{Indicadores para medir el grado de implicación del personal bibliotecario}

4.4.1. Porcentaje de personal formador del total de personal de la biblioteca en disposición de formar

En el siguiente gráfico se presenta la situación del personal bibliotecario que ha participado en el proyecto según categorías profesionales. 


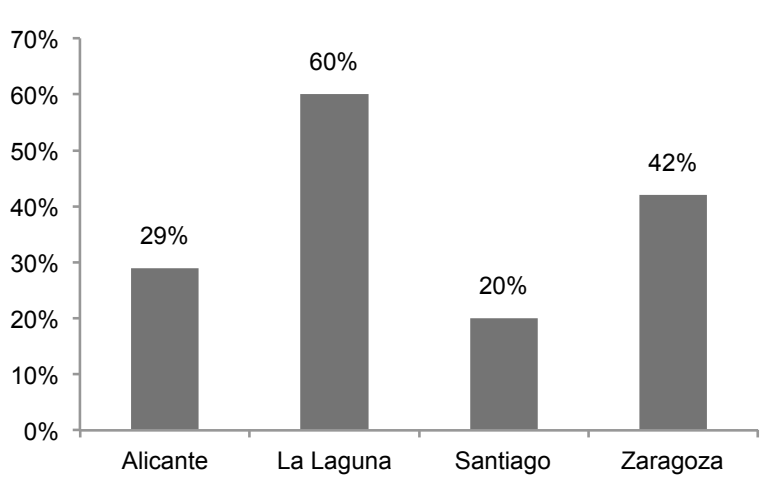

Gráfico VII. Implicación del personal bibliotecario

4.5. Indicadores para medir la satisfacción de los agentes implicados (alumnos, profesores, bibliotecarios)

En esta primera fase contamos con datos conjuntos sobre la satisfacción de los alumnos. La encuesta consta de doce ítems. Seleccionamos a continuación cuatro de los más significativos para medir la satisfacción con la actividad realizada

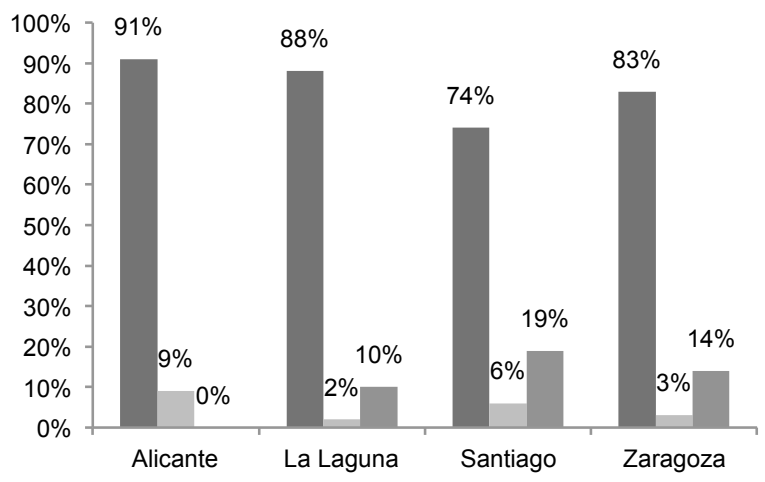

Gráfico VIII. Cumplimiento de los objetivos del curso

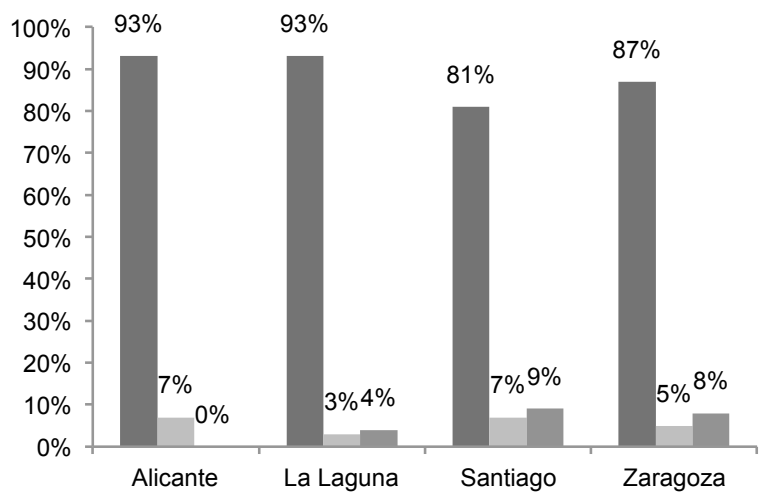

Gráfico IX. Conocimientos y habilidades aprendidas

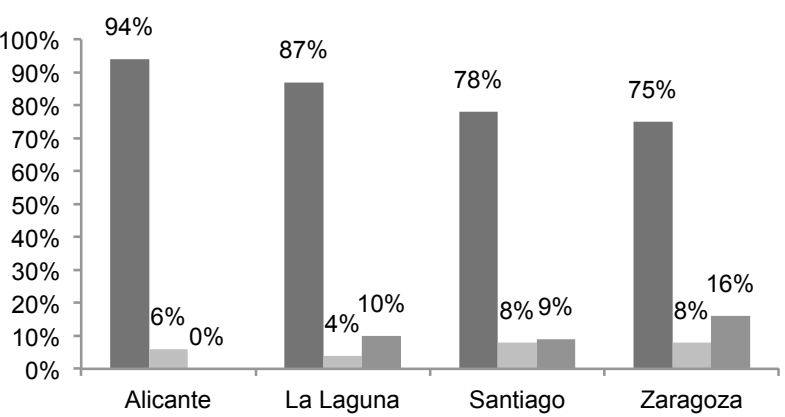

Gráfico X. Utilidad del curso

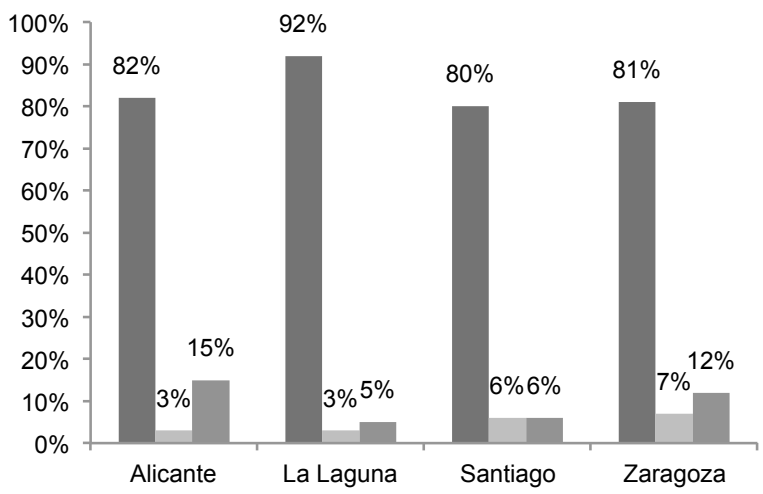

Gráfico XI. Satisfacción general del alumno

Uno de los objetivos para el curso 2011-12 es recopilar datos de satisfacción de personal bibliotecario formador, en base a la utilización de un formulario común, así como generalizar el uso del formulario de satisfacción para el profesorado.

\section{Difusión, impacto y futuro}

Esta experiencia arranca en común en el curso 2010-11 y en principio se toma como un proyecto piloto hasta la obtención de los primeros resultados comparables. Se adopta, como se ha dicho el modelo de la ULL con pequeñas excepciones por parte de la UZ y la UA, pero estableciendo desde el principio una serie de elementos comunes que permitiesen realizar posteriormente el análisis comparativo de resultados.

Se consiguió cumplir los objetivos marcados consolidándose el proyecto y poder iniciar el planteamiento de acciones futuras determinado un reparto de tareas y una responsabilidad concreta por universidad.

\subsection{Plan de difusión}

A la USC le fue encomendado el diseño y puesta en marcha del plan de difusión del proyecto. 
En primer lugar se consensuó la marca identificativa de la red de cooperación que nos distinguirá en todas las acciones que se lleven a cabo en la posteridad.

En segundo lugar se establecen dos estrategias para difundir el proyecto.

\subsubsection{Difusión interna}

Cada universidad tomará sus propias decisiones según sus expectativas, pero desde la USC se sugieren las siguientes acciones:

- Diseño de un banner anuncio para exponer en las web de las bibliotecas de las Universidades y en la web de cada centro o grado en el que se incorpore el curso.

- Organización de jornadas o seminarios compartidos entre bibliotecarios y profesores para dar a conocer más y mejor el modelo de curso y la experiencia de cooperación. En la Universidad de Santiago (campus de Santiago y Lugo) se celebra en el mes de junio uno de estos seminarios desde el Servicio de Planificación y Formación de PAS y desde el Programa de Formación e Innovación Docente (PFID) denominado Xornada sobre boas practicas na formación en competencias informacionais con la asistencia de más de 50 profesores y 30 bibliotecarios. El seminario fue impartido por las coordinadoras de los grupos de trabajo de las cuatro universidades conveniadas. El grado de satisfacción global en escala de 1 a 5 la media superó el 4.

Esta iniciativa está prevista que se extienda al resto de las universidades del convenio.

\subsubsection{Difusión externa con las siguientes accio-} nes:

- Elaboración de un video con la participación de profesores, alumnos y bibliotecarios para difundir en red la importancia de la adquisición de la competencia informacionales.

- Comunicación como buena práctica en las IX Jornadas CRAI (Santiago de Compostela 2011) con la presentación de resultados y evaluación comparativa del curso 2010/2011 de las cuatro bibliotecas universitarios del convenio. http://www.usc.es/es/congresos/ crai/index.html, dicha comunicación se publicó en la red Slideshare http://www.slideshare .net/CarmenVarelaPrado/uni-ci2-cooperacion -para-laformacion-en-competencias-modo-de -compatibilidad-8423699.

- Difusión del proyecto y del convenio de cooperación en los siguientes foros y redes sociales: IweTel, BibCat, Facebook, LinKedin, alfiniberoamerica.blogspot, briefinggalego y Alfared en la sección de buenas prácticas (evaluación)

\subsection{Impacto}

La repercusión que tuvo tanto la difusión del curso como el propio proyecto de cooperación llegó a sorprender al propio equipo de trabajo. Es verdad que el proyecto traspasó todas las fronteras y además de las felicitaciones que se llegaron a recibir, el impacto comenzó a materializarse en hechos concretos que analizamos de la misma forma que la difusión:

- Impacto interno en cada universidad. La Laguna -que ya tenía incorporado el curso en el $80 \%$ de los grados en el curso $2010 / 201-$ puede fácilmente acercarse al $90 \%$ en 2011 12 , Zaragoza de un $50 \%$ prevé alcanzar un $80 \%$ y Santiago del $13 \%$ llegará al $60 \%$.

- En cuanto al impacto externo, se mide por las propuestas de adhesión al convenio como nuevos asociados, estando ya incorporada la Universidad de Huelva y alguna otra en trámites.

- Desde Hispanoamérica, ya se han recibido también muestras de interés por el conocimiento más detallado del curso y los principios por los que se rige el convenio.

\subsection{El futuro}

No cabe la menor duda de que ante la experiencia vivida hasta el momento y los resultados obtenidos, en principio hay que destacar la actitud positiva e ilusionante que reina en el equipo.

Con el entusiasmo puesto en el trabajo, el futuro está orientado a la extensión de las competencias informacionales al mayor número de alumnos de primero de grado ante la necesidad de su adquisición, cuando entre el 85 y 90\% acceden a la universidad sin esta formación.

Como hemos ido exponiendo, se trabajan competencias informacionales pero teniendo en cuenta, que el modelo Crue/Tic/Rebium es el $\mathrm{Cl} 2$ (competencias informáticas e informacionales), y es un objetivo del equipo ir hacia este modelo trabajando ambas competencias conjuntamente.

Otro de los objetivos es mejorar el modelo con las aportaciones de todos las bibliotecas asociadas en el reparto de tareas y responsabilidades que ya hemos mencionado.

Así, la ULL, responsable de contenidos, actividades y de los cuestionarios de evaluación de 
las competencias, ha diseñado para este curso un nuevo modelo de cuestionario inicial y final que nos permita medir el aprendizaje del alumno, controlar desviaciones y replantear ítems. Por otra parte, se han simplificado contenidos y tareas adaptándolas y haciéndolas más comprensibles para el alumno.

La UA se ha responsabilizado de la elaboración de tutoriales y objetos de aprendizaje que faciliten la comprensión de los contenidos y ayude al estudiante en la elaboración de sus tareas: tutorial de operadores, uso de internet, estilos y modelos de citas y referencias bibliográficas.

La UZ, se ha encargado -y será su tarea fundamental- mejorar la tabla de indicadores, definiendo cada uno de ellos para evitar errores; establecer los criterios para el apto y no apto del alumno en el curso; los criterios que el profesor colaborador podrá utilizar para incorporar el curso en su guía docente; los cuestionarios de satisfacción de los alumnos, profesores y formadores; así como los indicadores con los que el profesor podrá aportar el rendimiento del alumno y mostrar la eficacia del curso.

Estos son los compromisos de cada biblioteca universitaria. Sin embargo hay que tener en cuenta que aunque jugamos en un mismo marco educativo, cada universidad tiene sus peculiaridades dadas por la propia autonomía. Hemos sido conscientes de ello y hemos planteado que, ante los debates que en ocasiones se suscitan sobre algunos aspectos, establecer unos mínimos que deben de ser respetados por todos y que van a ser los elementos evaluable en común.

Ante esto, se baraja ya la idea de elaborar un documento marco como manual del sistema, con los principios, objetivos, contenidos, procesos, procedimientos e indicadores comunes, y que nos lleve a determinar el perfil competencial del alumno de acuerdo al nivel exigible en primero de grado.

\section{Conclusión}

Hemos comprobado que trabajar juntos nos ayuda a mejorar de forma continua, nos da visibilidad, y nos permite hacer un estudio más amplio de la realidad en nuestras universidades y de la realidad de su alumnado.

El primer año de trabajo en común nos ha obligado a tener una comunicación permanente, que hemos hecho tanto de forma presencial como virtual (a través de un aula específica para el convenio).
De esta red de cooperación, con denominación propia UniCl2, destacamos como conclusión y a modo de resumen, sus principios y sus señas de identidad:

- Es una alianza, porque hemos establecido unos acuerdos de colaboración que se recogen en el convenio firmado y hemos creado una metodología de trabajo interno, con reparto de tareas y calendario de trabajo.

- Es una realidad porque hablamos de una experiencia real. La Laguna comenzó esta andadura en el año 2006, en el 2009 se inició el trabajo en equipo y Santiago, Zaragoza y Alicante han puesto en marcha este proyecto en el curso 2010-11.

- Es un modelo formativo concreto basado en la igualdad de oportunidades para todos los alumnos de nuevo ingreso. Esto se concreta en la oferta de un curso de formación en competencias a todos los alumnos de primero, con una actividad formativa asociada a una asignatura obligatoria y en colaboración con el profesor de la misma. La biblioteca participa así en la adquisición de las competencias informacionales, aunque la responsabilidad última sobre esta cuestión siempre es del profesor y del coordinador del Grado

- Se imparte en modalidad de enseñanza virtual con una pequeña sesión presencial y solamente requiere entre 5 y 10 horas de dedicación por parte del alumno, que puede hacerlo a su ritmo y sin interferir con otras tareas de su grado.

- Hemos establecido unos contenidos mínimos que garantizamos en todos los cursos de las bibliotecas del convenio. (Se ha tomado como base el documento sobre competencias informáticas e informacionales $\mathrm{Cl} 2$ elaborado en el seno de la Comisión Mixta CRUE-TICREBIUN.) Por otro lado, cada universidad tiene la libertad de ampliar estos mínimos cuanto quiera.

- Evaluamos y obtenemos resultados, para ello se han establecido indicadores y criterios de evaluación comunes de acuerdo con los contenidos mínimos. Esto nos permite comparar resultados.

- Es un objetivo estratégico para las bibliotecas participantes pero también para las universidades

Es visible y exportable. Trabajando juntos, autoridades académicas, profesorado, bibliotecarios, aunando esfuerzos y sinergias para seguir avanzando con el objetivo de formar personas competentes informacionalmente. 


\section{Recordatorio}

Las autoras de esta ponencia quieren recordar en ella a todos/as los compañeros/as formadores que han hecho posible esta realidad en todas y cada una de las universidades firmantes del convenio: ULL, USC, UZ, UA. Sin su trabajo no sería posible.

\section{Notas}

(1) En junio de 2011 se ha añadido como addenda la incorporación de Alicante

(2) La mayor parte de las bibliotecas integrantes utilizan la plataforma de enseñanza y aprendizaje Moodle.

(3) Este objetivo es fundamental puesto que la actividad de formación en competencias se inserta dentro de la actividad de aprendizaje que incluye la guía docente. Incluir este curso dentro de la guía es tarea fundamental para garantizar que todos los alumnos de primer curso tengan asegurada la formación.

(4) Claúsula Tercera del Convenio específico de colaboración entre las universidades de La Laguna, Santiago de
Compostela y Zaragoza para el desarrollo de un modelo de curso en competencias informacionales para el grado en el ámbito de las bibliotecas universitarias.

(5) En la USC el cálculo de la tasa de rendimiento se hizo sobre el total de alumnos matriculados en las asignaturas colaboradoras no sobre los alumnos inscritos en el curso. Sobre los alumnos inscritos, supone el $76 \%$.

\section{Referencias}

Competencias Informáticas e Informacionales en los estudio de Grado. Comisión mixta Crue/Tic/Rebiun, 2009. http://www.rebiun.org/doc/documento_competencias_inf ormaticas.pdf (2011-07-18).

Universidade de Santiago de Compostela (2010). Acordo do Consello de Gobernó polo que se establecen competencias susceptibles de recoñecemento para todas as titulacións de grao da USC como competencias transversais informacionais. Santiago de Compostela: Universidade, Consello de Gobernó, 2010. http://www.usc. es/export/sites/default/gl/normativa/descargas/acoconco mrectitigraocontran.pdf (2011-07-18). 
